\title{
Investigação teórica do processo de redução de coliformes em leitos cultivados
}

\author{
$\overline{\text { Mariângela Amendola }^{1} \text { \& Anderson L. Souza }}{ }^{1}$
}

\begin{abstract}
RESUMO
Este artigo mostra a continuidade da pesquisa sugerida por Amendola et al. (2003), para o caso em que se quer buscar maior precisão na investigação teórica do processo de redução de poluentes, em função do meio suporte usado em sistemas de leitos cultivados. Esta investigação se dá via modelagem matemática e simulação numérica do processo descrito por uma equação diferencial parcial de difusão-advecção, que envolve constantes que caracterizam o meio suporte (Moreno-Grau, 1996) e deve ser associada a condições iniciais e de contorno, estabelecidas de acordo com um procedimento experimental previamente realizado. Para resolução da equação implementa-se um algoritmo baseado no esquema implícito do método de diferenças finitas que, executado no MATLAB 6.1, gera valores da concentração dos poluentes que dependem dos valores das referidas constantes. Os resultados obtidos da simulação são comparados com valores de referência para a determinação do valor do coeficiente de dispersão longitudinal que, por sua vez, gera o menor resíduo para cada tipo de meio suporte do leito cultivado. Os resultados obtidos levam à conclusão de que o leito cultivado com brita e pneu picado é mais eficiente que aquele só com brita na remoção de coliformes fecais e totais.
\end{abstract}

Palavras-chave: simulação numérica, esquema implícito, redução de poluentes, problemas inversos

\section{Theoretical investigation of coliform reduction in constructed wetland}

\begin{abstract}
This paper shows the continuity of the research, as suggested by Amendola et al. (2003), when looking for better accuracy in the theoretical investigation of the pollutant reduction as a function of the support bed used in constructed wetland. This investigation is performed through the mathematical modeling and numerical simulation of the process, which is described by an advection-diffusion differential equation. This equation depends on certain constants which characterize the support bed (Moreno-Grau, 1996), and must be associated to the initial and boundary conditions established according to the experimental procedure. To solve the equation an algorithm based on the implicit scheme of the finite difference method was implemented which, when ran with MATLAB 6.1, generated values for the pollutant concentration which depend on the mentioned constants. The results obtained with the simulation were compared with the reference values to determine the longitudinal dispersion coefficient value which leads to the least residual value for each kind of support bed. The obtained results lead to the conclusion that the constructed wetland with crushed stone and chopped tires is better than the one with crushed stone alone to reduce fecal and total coliforms.
\end{abstract}

Key words: numerical simulation, implicit scheme, inverse pollutant reduction, inverse problems 


\section{INTRODUÇÃO}

De acordo com a Agenda 21 (1996) “estima-se que 80 por cento de todas as moléstias e mais de um terço dos óbitos dos países em desenvolvimento sejam causados pelo consumo de água contaminada e, em média, até um décimo do tempo produtivo de cada pessoa se perde devido a doenças relacionadas com a água”.

Essas estimativas, que pouco mudaram desde o século passado, mostram a importância da realização de pesquisas que levem à proposição de tecnologias capazes de minimizar as agressões sofridas por este recurso natural como, por exemplo, tecnologias para o tratamento de águas residuárias.

Devido às suas características, os sistemas naturais de tratamento de águas residuárias têm, em geral, baixo custo de construção e operação associado a uma alta eficiência, o que atende a uma das recomendações da Agenda 21 (1996), segundo a qual se deve buscar, sempre que possível, meios tecnológicos e científicos de baixo custo; neste sentido, se verificam, no Brasil, nos últimos anos, grandes avanços em pesquisa e desenvolvimento de processos e técnicas de tratamento de águas residuárias, direcionados tanto para sistemas de grande porte e de maior complexidade, quanto de pequeno porte, baixo custo e simplicidade operacional, tais como tanques sépticos, lagoas de estabilização, reatores anaeróbios, disposição nos solos e leitos cultivados (Mazzola, 2003).

Em relação aos leitos cultivados (ou constructed wetlands), talvez sejam esses os sistemas ainda relativamente pouco estudados no Brasil, ao menos na exploração de todo o seu potencial (Mazzola et al., 2005).

Em muitas das investigações experimentais envolvendo a construção de leitos cultivados, como as realizadas na FEAGRI/UNICAMP, se tem optado pelo cultivo da macrófita Typha sp., por serem abundantes e bem adaptadas à região de Campinas e, como meio suporte, pela utilização de pedra britada (Valentim, 1999; Hussar, 2001; Tobias, 2002), ou pedra britada e pneu picado (Collaço, 2002), caso em que se considera que a utilização de materiais alternativos é uma medida que pode baratear ainda mais os sistemas de leitos cultivados além de lhes servir como destino final e assim trazer benefícios adicionais ao meio ambiente.

Dando continuidade a este tipo de investigação, porém indo além uma vez que, também usando de ferramentas teóricas, foram realizados vários tipos de análises qualitativas, via modelagem matemática e simulação numérica do processo dinâmico da redução de poluentes em leitos cultivados (Valentim \& Amendola, 1999; Amendola \& Ladeira, 2000; Amendola \& Souza, 2002; Amendola et al., 2003), sempre a partir do modelo matemático proposto por Moreno-Grau (1996).

Amendola et al. (2003) apresentam resultados da simulação numérica do referido processo dinâmico para a subseqüente análise qualitativa da eficiência dos dois tipos de meio suporte, citados anteriormente, na remoção de coliformes fecais em leitos cultivados com Typha sp., e sugerem o uso da ferramenta de análise de resíduos, para a análise quantitativa associada.

Finalmente, na dissertação de mestrado de Souza, 2003, se encontram, entre outros resultados experimentais que permitem a realização deste tipo de análise quantitativa, os resulta- dos da análise de influência dos dois tipos de meio suporte, pedra britada e pneus picados, no processo de remoção de coliformes fecais e coliformes totais, em leitos cultivados com Typha sp., que são os resultados apresentados neste artigo.

Dentre os objetivos desta pesquisa se destacam: a determinação do valor da constante de dispersão, que surge como um dos fatores do coeficiente de dispersão longitudinal da equação diferencial parcial de advecção-difusão do modelo matemático proposto por Moreno-Grau (1996); a avaliação do desempenho de cada um dos dois tipos de meio suporte, pedra britada e pneus picados, no processo de remoção de coliformes fecais e coliformes totais em leitos cultivados com Typha sp..

\section{MATERIAL E MÉTODOS}

Os leitos cultivados foram construídos no Centro Avançado de Pesquisa Tecnológica do Agronegócio de Engenharia e Automação (CAPTA - Engenharia e Automação), antigo Centro de Mecanização e Automação Agrícola (CMAA), do Instituto Agronômico de Campinas (IAC), localizado no município de Jundiaí, SP/BR (23 10’ 51” S, 4657’00” W).

$\mathrm{O}$ esgoto gerado dentro do CAPTA - Engenharia e Automação, é coletado e enviado para uma Estação de Tratamento de Esgoto (ETE) dentro do próprio centro, formada por uma grade, para retenção dos sólidos mais grosseiros, seguida de um tanque de aeração no qual ocorre degradação da matéria orgânica, por meio de bactérias aeróbias, e de um decantador, onde se dá a sedimentação dos sólidos suspensos; após este pré-tratamento, parte do efluente da ETE recebe um polimento e é lançada no córrego Herminda, um afluente do Rio Jundiaí, enquanto outra parte é bombeada para um tanque de equalização, que é uma caixa de cimento de amianto de $1000 \mathrm{~L}$ onde ocorre a homogeneização do efluente e a partir daí, pela ação da gravidade e através de tubos de PVC com 25 mm de diâmetro, segundo um mecanismo de distribuição, segue para os dois leitos cultivados de fluxo subsuperficial.

Esses leitos foram construídos paralelamente, acima do nível do solo, em alvenaria de blocos de cimento de 0,2 x 0,4 m, com base de concreto armado e uma inclinação inferior a 1\%. Cada um dos leitos tem $5 \mathrm{~m}$ de comprimento, $2 \mathrm{~m}$ de largura e $1 \mathrm{~m}$ de altura. $\mathrm{O}$ revestimento interno dos tanques foi feito com argamassa e impermeabilizante.

Um dos leitos, denotado por LC1, foi completamente preenchido com brita $\mathrm{n}^{\circ} 3$ (55 a 90 mm de diâmetro), e o outro leito, denotado por LC2, foi preenchido com pneu picado, até aproximadamente $0,80 \mathrm{~m}$ de altura e o restante $0,20 \mathrm{~m}$ com brita n. 3 .

O pneu picado utilizado como meio suporte se originou de um lote em que estavam misturados pneus de trator, caminhão e automóveis passados por um triturador de pneus, da marca Satren Italiana, regulado com grelha de 6 polegadas.

Devido à constituição do pneu, investigou-se a possibilidade do pneu picado liberar metais pesados para o esgoto, através de análises realizadas nos laboratórios especializados, como a Central Analítica (CA) do Instituto de Química (IQ) da UNICAMP.

Manteve-se a coluna d’água abaixo do meio suporte, a 
0,9 m de altura; desta forma e uma vez determinados os volumes de vazios, é possível calcular o volume útil dos leitos e, fixando-se o tempo de detenção hidráulica dos leitos em dois dias, fazer o cálculo das respectivas vazões.

Uma vez conhecidas as vazões do leitos regulou-se o registro do mecanismo de distribuição, de forma a manter essas vazões fixas durante todo o período experimental; ambos os leitos, LC1 e LC2, foram cultivados com aproximadamente 40 mudas de Typha sp., popularmente conhecida como taboa.

Nota-se que a floresta de pinheiros, instalada nas proximidades da ETE para evitar problemas de odor, não só causou um sombreamento diferenciado nos leitos, mas levou à morte de algumas plantas e ao replantio de mudas nos mesmos, sugerindo, também, o monitoramento da sua tempeatura.

Estabelecidas as condições do procedimento experimental, cujos detalhes se encontram em Souza (2003), procedeu-se à coleta de dados especificados, ou seja, diariamente, no período de 10 a 16 de julho de 2002, foram coletadas amostras das concentrações dos poluentes coliformes totais e fecais, tanto dos afluentes, com velocidades u $\left(\mathrm{LC}_{1}\right)=1,094\left[\mathrm{~m} \mathrm{dia}^{-1}\right]$ e u $\left(\mathrm{LC}_{2}\right)=1,446$ [m dia-1], como dos efluentes do $\mathrm{LC}_{1}$ e do $\mathrm{LC}_{2}$, e foram tomados, como valores de referência, os dados de determinado dia e os dados após 2 dias de detenção; esses valores de referência são os valores da concentração dos poluentes referentes às condições iniciais e de contorno, cujos valores numéricos serão especificados ao longo do texto.

As amostras foram submetidas a análises laboratoriais realizadas no Laboratório de Saneamento da FEAGRI, para determinação das concentrações, dentre outros, dos poluentes coliformes total e coliformes fecais, pelo método cromogênico, através da cartela Quanti-Tray 2000, seladora QuantiSealer e lâmpada UV.

Para descrever matematicamente o processo em questão, considerou-se o modelo matemático proposto por MorenoGrau (1996), escrito para a concentração de cada um dos poluentes ao longo dos leitos e a cada tempo, $\mathrm{C}=\mathrm{C}(\mathrm{x}, \mathrm{t})$, já utilizado para descrever também o caso em que o coeficiente de dispersão longitudinal é considerado constante ao longo de leitos de fluxo superficial:

$$
\frac{\partial C}{\partial t}=D_{x}\left(\frac{\partial^{2} C}{\partial x^{2}}\right)-u \frac{\partial C}{\partial x}+\sum_{z=1}^{n p} r_{z} ; t \geq 0 \text { e } x \in\left[0, C_{L}\right]
$$

donde:

$$
\begin{aligned}
& \text { C - concentração do poluente, NMP/100 mL } \\
& \mathrm{t} \text { - tempo, dia } \\
& \mathrm{x} \text { - direção longitudinal, } \mathrm{m} \\
& \mathrm{r}_{\mathrm{z}} \text { - termo referente a cada um dos processos físicos, } \\
& \text { químicos ou bioquímicos, mg } \mathrm{L}^{-1} \mathrm{dia}^{-1} \\
& \mathrm{n}_{\mathrm{p}} \text { - número total de processos } \\
& \mathrm{u} \text { - velocidade média do afluente na direção } \mathrm{x}, \mathrm{m} \text { dia }^{-1} \\
& \mathrm{C}_{\mathrm{L}} \text { - comprimento do leito, } \mathrm{m} \\
& \mathrm{D}_{\mathrm{X}}-\text { coeficiente de dispersão longitudinal, } \mathrm{m}^{2} \mathrm{dia}^{-1} \text {, }
\end{aligned}
$$

$$
\mathrm{D}_{\mathrm{x}}=3,134 \mathrm{~K} \mathrm{n} \mathrm{u} \mathrm{d}^{6 / 5}
$$

em que:
3,134 - constante empírica do modelo, adimensional

$\mathrm{K}$ - constante de dispersão, adimensional

$\mathrm{n}$ - coeficiente de rugosidade de Manning, adimensional

$\mathrm{u}$ - velocidade média do afluente, $\mathrm{m}$ dia $^{-1}$

$\mathrm{d}$ - profundidade do leito, $\mathrm{m}$

Na Eq. 1 o primeiro termo do lado direito representa o processo de difusão do poluente, enquanto o segundo se refere ao processo de sua advecção, devido à velocidade u, e o terceiro termo, os demais fenômenos responsáveis pela variação da concentração, ao longo do espaço e tempo.

Neste estudo, em que os leitos são de fluxo subsuperficial, C representa adequadamente a concentração de coliformes totais ou fecais, $\mathrm{z}=1$, e

$$
\mathrm{r}_{1}=-\mathrm{K}_{\mathrm{ef}} \mathrm{C}
$$

sendo:

$\mathrm{K}_{\mathrm{ef}}$ - constante cinética de mortalidade dos coliformes, dia $^{-1}$.

Ainda assim, são considerados os seguintes valores para as constantes e parâmetros do modelo já utilizados por MorenoGrau (1996): $K_{\mathrm{ef}}=4,0 \mathrm{dia}^{-1}$ e $\mathrm{n}=0,025$. Nesta investigação, portanto, o parâmetro cujo valor ainda pode ser investigado, de maneira a determinar o valor do coeficiente de dispersão longitudinal e então caracterizar a influência do meio suporte no leito cultivado no processo de remoção de poluentes, é a constante de dispersão $\mathrm{K}$, ante o que, para se comparar a eficiência dos leitos no processo de remoção de poluentes deve-se notar que um leito é mais ou menos eficiente que outro quanto menor ou maior for o valor da constante $\mathrm{K}$ a ele associado.

Os valores de referência são as condições iniciais e de contorno que devem ser associadas à Eq. 1; desta forma, para a concentração de coliformes fecais considerou-se:

$$
\begin{aligned}
& \mathrm{C}(0, \mathrm{t})=25950 \text { e } \mathrm{C}\left(2 \mathrm{C}_{1}, \mathrm{t}\right)=0 ; \forall \mathrm{t} \geq 0 \\
& \mathrm{C}(\mathrm{x}, 0)=25950 ; \mathrm{x} \in\left[0, \mathrm{C}_{1}\right] ; \mathrm{C}\left(\mathrm{C}_{1}, 0\right)=0
\end{aligned}
$$

enquanto para coliformes totais se considerou:

$$
\begin{aligned}
& C(0, t)=173290 \text { e } C\left(2 C_{1}, t\right)=0 ; \forall t \geq 0 \\
& C(x, 0)=173290 ; x \in\left[0, C_{1}\right] ; C\left(C_{1}, 0\right)=0
\end{aligned}
$$

Nota-se, em ambos os casos, que uma das condições de contorno é tomada em $2 \mathrm{C}_{\mathrm{l}}$, que é a estratégia para evitar o efeito das condições de contorno.

Para a simulação numérica de $C=C(x, t)$ segue-se o seguinte conjunto de passos:

i) discretiza-se a região espacial e o domínio do tempo em estudo em Nx e Nt subintervalos respectivamente;

ii) considera-se a convenção:

$$
\mathrm{C}_{\mathrm{i}}^{\mathrm{n}}=\mathrm{C}(\mathrm{i} \Delta \mathrm{x}, \mathrm{n} \Delta \mathrm{t}) ; \mathrm{i}=1, \ldots \mathrm{Nx} \text { e } \mathrm{n}=0, \ldots \mathrm{Nt}
$$

em que $\Delta$ t é o passo de integração no tempo e $\Delta x$ é o tamanho da malha espacial; e

iii) para as derivadas que aparecem na Eq. 1, selecionamse as seguintes aproximações com aproximação da ordem de $\Delta \mathrm{t}, \Delta \mathrm{x}^{2}$ e $\Delta \mathrm{x}$, respectivamente: 


$$
\begin{aligned}
& \frac{\partial C^{n}}{\partial t_{i}} \cong\left(C_{i}^{n+1}-C_{i}^{n}\right) / \Delta t \\
& \frac{\partial^{2} C^{n}}{\partial x_{i}^{2}} \cong\left(C_{i-1}^{n+1}-2 C_{i}^{n+1}+C_{i+1}^{n+1}\right) / \Delta x^{2} \\
& \frac{\partial C^{n}}{\partial x_{i}} \cong\left(C_{i}^{n+1}-C_{i-1}^{n+1}\right) / \Delta x
\end{aligned}
$$

As condições iniciais e de contorno também são discretizadas segundo a convenção estabelecida.

A partir dessas especificações, a Eq. 1 fica expressa para o cálculo de $\mathrm{C}=\mathrm{C}(\mathrm{x}, \mathrm{t})$ de acordo com:

$$
\begin{aligned}
& -\left[\left(D_{x} f 2\right)+(u f 1)\right] C_{i-1}^{n+1}+\left[1+2\left(D_{x} f 2\right)+(u f 1)\right] C_{i}^{n+1} \\
& -\left(D_{x} f 2\right) C_{i+1}^{n+1}=\left(1-K_{e f}\right) \Delta t C_{i}^{n}
\end{aligned}
$$

donde

$$
\frac{\Delta \mathrm{t}}{\Delta \mathrm{x}}=\mathrm{fl} \text { e } \frac{\Delta \mathrm{t}}{\Delta \mathrm{x}^{2}}=\mathrm{f} 2
$$

Para cada instante de tempo n fixo, e se fazendo i variar de 1 até $\mathrm{N}_{\mathrm{x}}$, a Eq. 12 gera um sistema tridiagonal de equações lineares que, após ser resolvido, gera a concentração do poluente $\mathrm{C}$ naquele instante, para todo ponto da malha espacial.

Observa-se que este é o tipo de problema matemático caracterizado como sendo da classe dos problemas inversos (Campos Velho, 2001), para os quais existem técnicas numéricas mais robustas, uma vez que tal sistema pode ser mal condicionado mas cujo uso não é objeto desta investigação.

Para determinação do valor da constante de dispersão K em cada leito, foi implementado um algoritmo que, para cada um dos valores de $\mathrm{K}$ dentro de um intervalo estimado (Amendola et al., 2003), calcula os valores associados do coeficiente de dispersão longitudinal, $\mathrm{D}_{\mathrm{x}}$; este valor é, então, utilizado no algoritmo préestabelecido pelo uso do esquema ímplícito do método numérico de diferenças finitas e associado ao método de Crout, para a resolução do sistema e, em seguida, o algoritmo busca o valor que gera menor valor residual da comparação entre os dados de concentração então simulados e os respectivos dados experimentais de referência, no ponto da malha correspondente a $5 \mathrm{~m}$.

A implementação do algoritmo assim composto, foi realizada com o ambiente de computação científica MATLAB 6.1.

\section{RESULTADOS E DISCUSSÃO}

Os resultados obtidos da análise de metais pesados confirmam a classificação apresentada em CETESB (2003), segundo a qual os pneus são classificados como resíduos inertes para efeito de disposição final, não havendo riscos de que seus componentes se solubilizem ou contaminem o sistema.

A temperatura da água apresentou um comportamento bastante semelhante em ambos os leitos, sendo que no LC1 a temperatura média foi de $18{ }^{\circ} \mathrm{C}$, com desvio padrão de $1,65^{\circ} \mathrm{C}$ e, no LC2, a temperatura média foi de $17,95^{\circ} \mathrm{C}$, com desvio padrão de $1,64^{\circ} \mathrm{C}$. Através de uma análise de vari- ância com base em um teste F, a nível de 5\% de significância, confirmou-se que não há diferença estatisticamente significante entre as temperaturas da água dentro dos leitos.

Antes de se iniciar a investigação da influência do meio suporte no processo de remoção dos poluentes, via simulação numérica do processo, realizou-se a análise da influência do tamanho da malha espacial até então não realizada com rigor, por Amendola et al. (2003); para tanto foram tomados, como exemplo, um valor fictício de $\mathrm{K}$ e as especificações de um dos leitos e de um dos poluentes; com essas especificações e mantendo todos os demais parâmetros constantes, simulou-se a concentração desse poluente para malhas cada vez mais finas e até que não se observasse diferença significativa entre resultados de malhas subseqüentes.

A Figura 1 mostra o erro relativo oriundo da comparação entre os resultados obtidos no ponto final do leito (5 m) para os distintos valores do número de pontos da malha espacial, desde 5 até 101; por outro lado se pode verificar, nesta figura, que a partir de 45 pontos não há diferença significativa entre os resultados simulados.

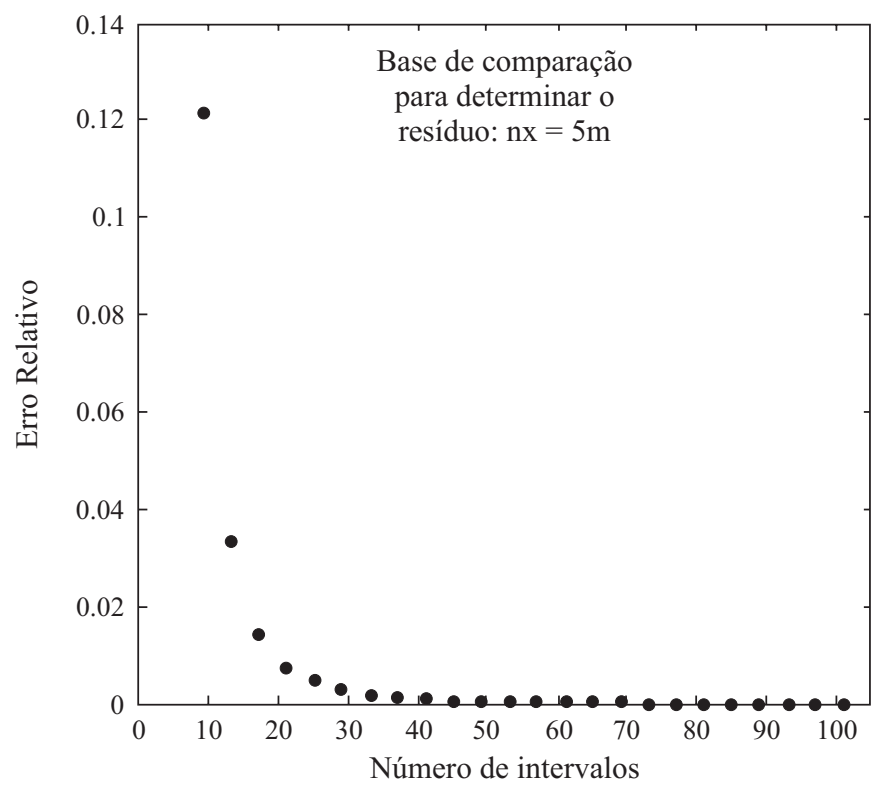

Figura 1. Erro relativo em função do número de pontos da malha, calculado no ponto final do leito correspondente à distância de $5 \mathrm{~m}$ da origem do leito

Observa-se que o tempo de execução do programa aumenta com o número de pontos da malha mas este não é significativo de vez que, por ser implícito, o programa gasta cerca de $0,01 \mathrm{~s}$ para a malha mais grossa (5 pontos) e cerca de $0,6 \mathrm{~s}$ para a malha mais fina (101 pontos).

Tendo em vista essas considerações foram usadas, em seguida, malhas com 45 pontos para realizar as distintas simulações da concentração de cada um dos poluentes para cada um dos leitos, variando-se o valor da constante de dispersão $\mathrm{K}$ em intervalos préestabelecidos (Amendola et al., 2003).

A Figura 2 indica os valores de coliformes totais simulados em $\mathrm{LC}_{1}$ para valores de $\mathrm{K}$ variando entre 10 e 300 e a Figura 3 mostra os resíduos calculados da comparação desses valores com os valores de referência.

Na Figura 2 o vetor indica o sentido de acréscimo do valor 


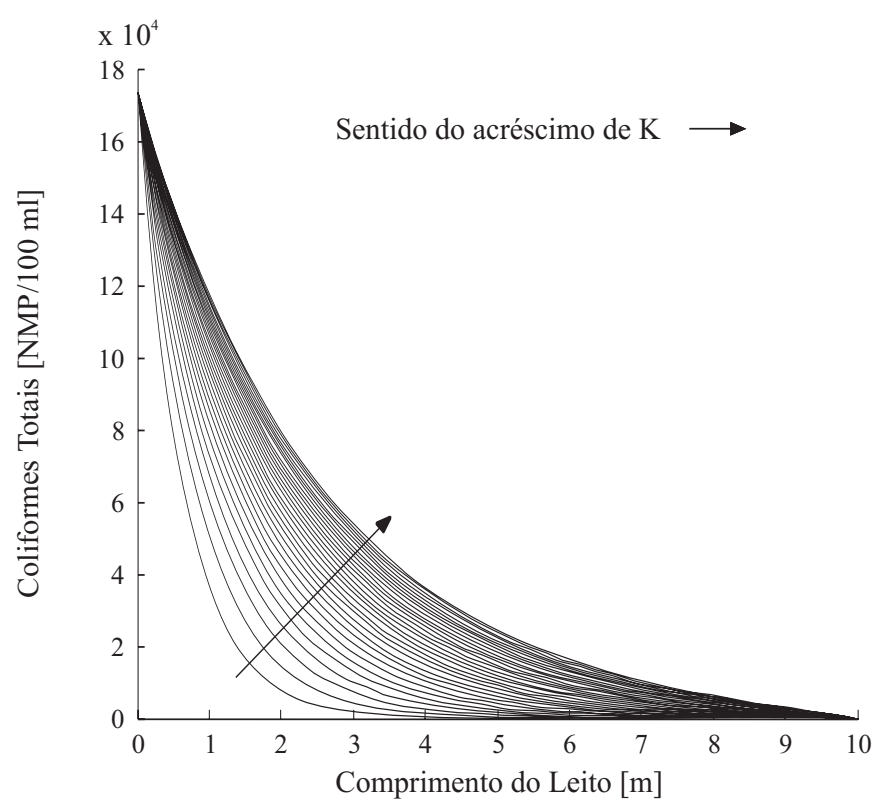

Figura 2. Concentração simulada de Coliformes Totais no $\mathrm{LC}_{1}$ para valores de $\mathrm{K}$ entre 10 e 300

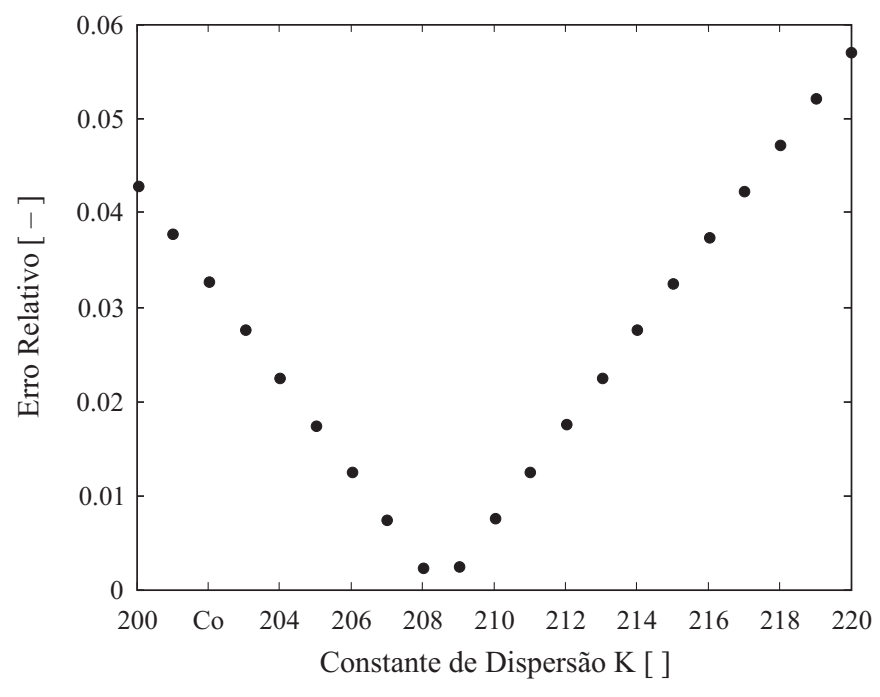

Figura 3. Erros relativos entre os valores obtidos numericamente e os valores de referência

de $\mathrm{K}$ e as linhas são contínuas apenas para facilitar a visualização enquanto na Figura 3 se pode verificar que o valor que apresenta menor resíduo está em torno de 209.

De maneira análoga, este procedimento foi realizado para o $\mathrm{LC}_{2}$ e, posteriormente, o foi feito também para o caso de coliformes fecais, tanto no $\mathrm{LC}_{1}$ quanto no $\mathrm{LC}_{2}$.

A Tabela 1 mostra o resumo dos resultados então obtidos para os casos de coliformes totais e coliformes fecais nos dois tipos de leito.

Tabela 1. Melhores valores de K [ ] para os casos de coliformes totais e coliformes fecais em cada um dos leitos

\begin{tabular}{ccc}
\hline \multirow{2}{*}{ Leito } & \multicolumn{2}{c}{ Melhor valor de $\mathrm{K}$} \\
\cline { 2 - 3 } & Coliformes totais & Coliformes fecais \\
\hline $\mathrm{CC}_{1}$ & 209 & 342 \\
$\mathrm{LC} \mathrm{C}_{2}$ & 46 & 40 \\
\hline
\end{tabular}

Ressalta-se que, quanto menor o valor de $\mathrm{K}$ associado a um tipo de leito menor, portanto, o valor de $D_{x}$, uma vez que os seus demais fatores foram mantidos constantes, melhor é a eficiência deste leito no processo de remoção de poluentes; notase, nos valores indicados na Tabela 1 , que o leito $\mathrm{LC}_{2}$ é o que apresenta melhor eficiência para os dois tipos de poluentes.

Devido à quantidade de fatores que não puderam ser controlados no procedimento experimental como, por exemplo, as condições climáticas, além da impossibilidade de repetições do procedimento e das análises das amostras e das fontes de erros dessas análises, das aproximações do modelo matemático e as limitações da metodologia numérica, não se pode concluir quanto à causa desses resultados sobre a eficiência dos leitos; mesmo assim e se considerando os melhores valores de $\mathrm{K}$ obtidos para cada um dos tipos de poluentes para os dois tipos de leito, como indicado na Tabela 1, tem-se a representação do processo de remoção dos poluentes em cada um dos tipos de leito, como indicam as Figuras 4 e 5; este processo de remoção deve ser observado pela redução dos níveis de

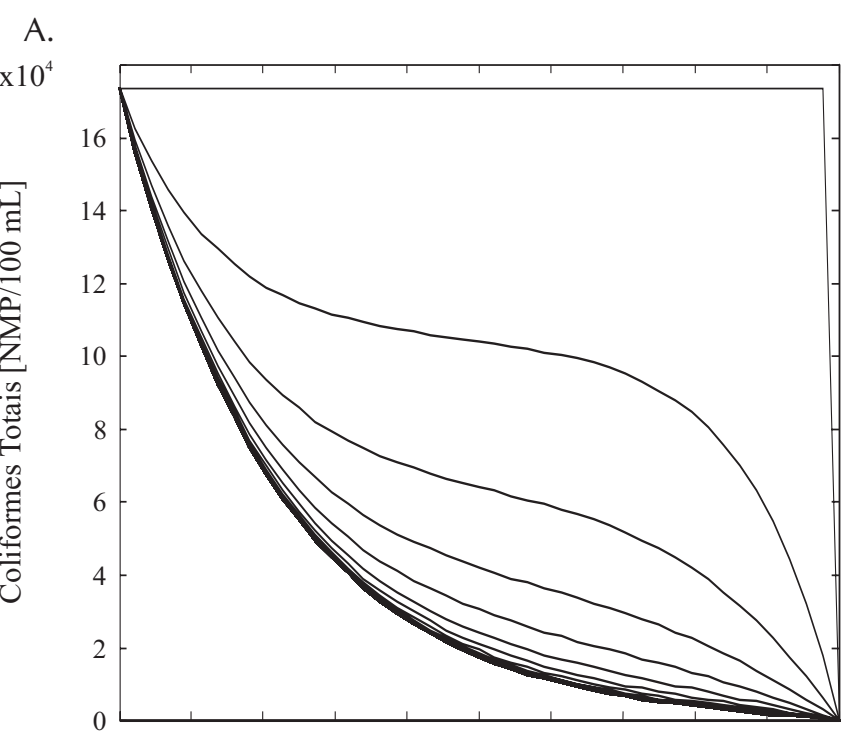

B.

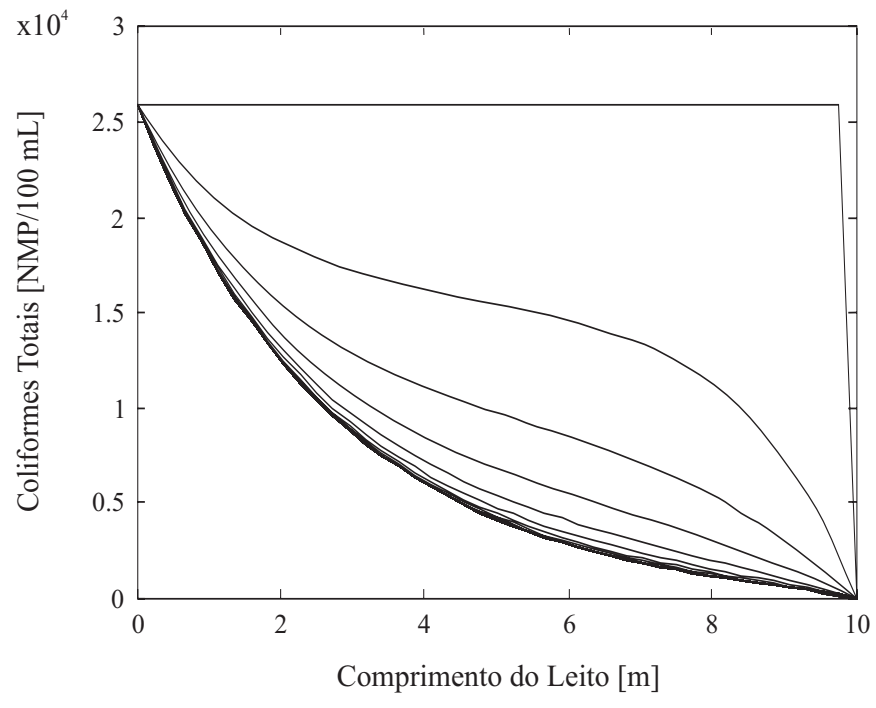

Figura 4. Simulação numérica da concentração em $\mathrm{LC}_{1}$ de coliformes totais (A) e fecais (B) ao longo do espaço em diversos instantes 


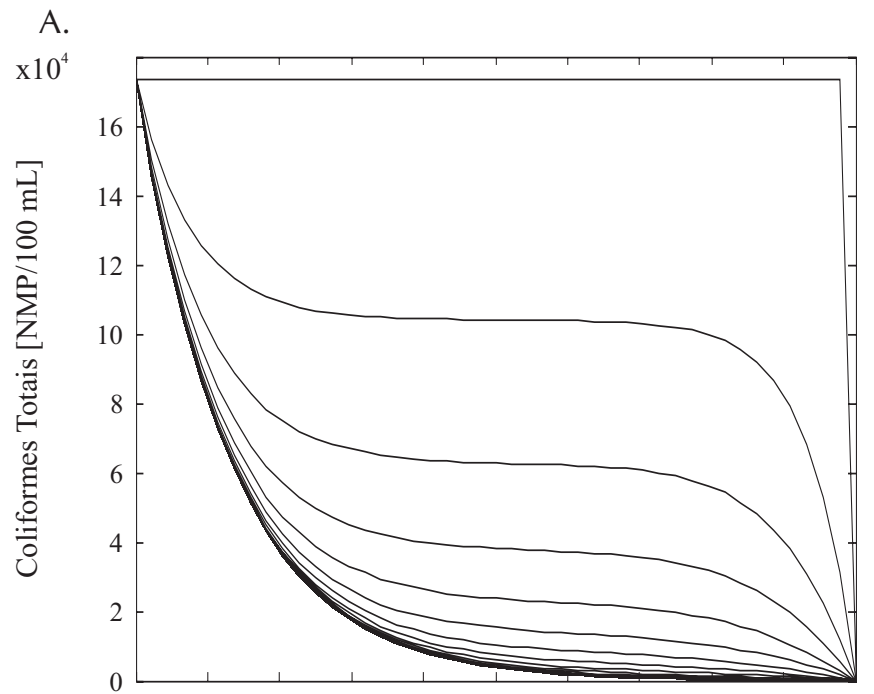

B.

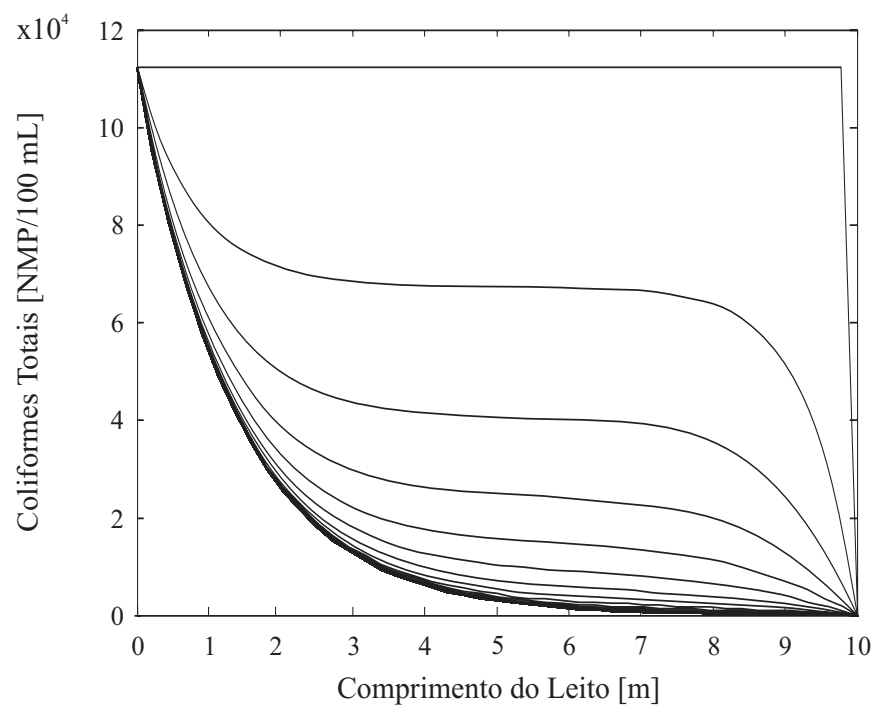

Figura 5. Simulação numérica da concentração em $\mathrm{LC}_{2}$ de coliformes totais (A) e fecais (B) ao longo do espaço em diversos instantes

concentração do poluente ao longo do espaço em diversos instantes de tempo selecionados; nessas figuras as curvas aparecem contínuas apenas para facilitar a visualização.

\section{CONCLUSÕES}

1. É possível incorporar, ao modelo matemático, a influência dos distintos tipos de meio suporte nos leitos cultivados o que contribuirá, sem dúvida, com o processo de discussão e tomada de decisão para a construção desse tipo de sistema natural de tratamento de esgotos.

2. Para o caso dos coliformes totais os valores ajustados para $\mathrm{K}$ foram 209 e 46 no $\mathrm{LC}_{1}$ e $\mathrm{LC}_{2}$, respectivamente, enquanto para o caso de coliformes fecais os valores ajustados para $\mathrm{K}$ foram 342 e 40 no $\mathrm{LC}_{1}$ e $\mathrm{LC}_{2}$, respectivamente.

3. O leito cultivado que apresentou melhor eficiência foi o que também constou do meio suporte misto de brita e pneu picado.
4. A repetição do experimento poderia gerar subsídios para uma discussão detalhada dos motivos que levaram ao tipo de leito a ter maior eficiência.

5. O desenvolvimento de uma metodologia específica para a resolução deste tipo de problema inverso poderia contribuir na redução de possíveis erros oriundos da metodologia numérica usada.

\section{AgRADECIMENTOS}

Os autores agradecem à Sociedade Brasileira de Matemática Aplicada e Computacional - SBMAC, pela oportunidade de discutir os resultados no CNMAC 2003, e ao FAEPEX/UNICAMP, pelo auxílio financeiro para a mesma oportunidade.

\section{LITERATURA CITADA}

Agenda 21. Proteção da qualidade e do abastecimento dos recursos hídricos: Aplicação de critérios integrados no desenvolvimento, manejo e uso dos recursos hídricos. Rio de Janeiro, 1996. http://www.agenda21.org.br/full/hidricos-full.htm. 23 Mar. 2005.

Amendola, M.; Ladeira, S. S. Numerical simulation of the total coliform reduction process in constructed wetlands cultivated with macrophytes. In: National Congress on Applied and Computational Mathematics, 23, 2000. Santos. Resumos... Santos: SBMAC, 2000. p.89.

Amendola, M.; Souza, A. L. Mathematical modeling and numerical simulation of the pollutants reduction at constructed wetland. In: Italian Congress of Computational Mechanics, 14, and Ibero Latin American Congress on Computational Methods for Engineering, 23-CILAMCE 2002, Giulianova. Resumos... Giulianova: ABMEC, 2002. CD Rom

Amendola, M.; Souza, A. L.; Roston, D. M. Numerical simulation of fecal coliform reduction at a constructed wetland. Revista Brasileira de Engenharia Agrícola e Ambiental, Campina Grande, v.7, n.3, p.533-538, 2003.

Campos Velho, H. F. Problemas inversos: Conceitos básicos e aplicações In: Encontro de Modelagem Computacional, 4, 2001, Nova Friburgo. Resumos... São Carlos: SBMAC, 2001. Mini-curso convidado, p.63-79.

CETESB - Companhia de Tecnologia de Saneamento Ambiental. Resíduos sólidos: Disposição de pneus em aterros. http:// www.cetesb.sp.gov.br/Solo/solo_pneus.htm. 12 Mar. 2003.

Collaço, A. B. Avaliação do uso de pneu picado como meio suporte de leitos cultivados para o tratamento de esgoto sanitário. Campinas: FEAGRI/UNICAMP, 2002. 123p. Dissertação Mestrado

Hussar, G. J. Avaliação do desempenho de leitos cultivados no tratamento de águas residuárias de suinocultura. Campinas: FEAGRI/UNICAMP, 2001. 118p. Dissertação Mestrado

Mazzola, M. Uso de leitos cultivados de fluxo vertical por batelada no pós-tratamento de efluente de reator anaeróbio compartimentado. Campinas: UNICAMP, 2003. 99p. Dissertação Mestrado 
Mazzola, M.; Roston, D. M.; Valentim, M. A. A. Post-treatment of effluent of anaerobic baffled reactor using batchwise vertical flow constructed wetland beds. Revista Brasileira de Engenharia Agrícola e Ambiental, Campina Grande, v.9, n.2, p.276-283, 2005.

Moreno-Grau, S. A. Mathematical model for wastewater stabilization ponds with macrophytes and microphytes. Ecological Modeling, v.91, p.77-103, 1996.

Souza, A. L. Estudo experimental e teórico do processo de remoção de poluentes nos leitos cultivados. Campinas: UNICAMP, 2003. 116p. Dissertação Mestrado
Tobias, A. C. T. Tratamento de resíduos da suinocultura: uso de reatores anaeróbicos seqüenciais seguido de leitos cultivados. Campinas: FEAGRI/UNICAMP, 2002. 125p. Tese Doutorado

Valentim, M. A. A. Uso de leitos cultivados no tratamento de efluente de tanque séptico modificado. Campinas: UNICAMP, 1999. 119p. Dissertação Mestrado

Valentim, M. A. A.; Amendola, M. Numerical simulation of the dynamics of $E$. coli reduction in constructed wetlands: A case study. In: Iberian-Latin American Congress on Computational Methods in Engineering, 20, 1999, São Paulo. Proceedings... São Paulo: ABMEC, 1999, CD Rom 